Citation: G. Scaglione (2021). Una rappresentazione diplomatica e di parte: il ritratto (geo)letterario della Tunisi di Guglielmo Collotti (1876). Bollettino della Società Geografica Italiana serie 14, 4(1): 39-52. doi: 10.36253/bsgi-1242

Copyright: ( $2021 \mathrm{G}$. Scaglione. This is an open access, peer-reviewed article published by Firenze University Press (http://www.fupress.com/bsgi) and distributed under the terms of the Creative Commons Attribution License, which permits unrestricted use, distribution, and reproduction in any medium, provided the original author and source are credited.

Data Availability Statement: All relevant data are within the paper and its Supporting Information files.

Competing Interests: The Author(s) declare(s) no conflict of interest.

\section{Una rappresentazione diplomatica e di parte: il ritratto (geo)letterario della Tunisi di Guglielmo Collotti (1876)}

\author{
A Diplomatic and Partisan Representation: the (Geo)-Literary \\ Portrait of Tunis by Guglielmo Collotti (1876)
}

\author{
Giannantonio Scaglione \\ Dipartimento di Lettere e Filosofia, Università degli Studi di Trento, Italia \\ E-mail: g.scaglione@unitn.it
}

\begin{abstract}
The paper aims to retrace the (geo)-literary portrait of the city of Tunis by analysing the work Tunisi e il suo popolo. Studi impressioni e ricordi (Tunis and its people. Studies, impressions and memories), written by Guglielmo Collotti and published in Catania in 1876. This work is a travel report of a journey planned by Collotti with the excuse of presenting a "philanthropic" award to the sovereign of the Regency of Tunis. In fact, it gave the Sicilian nobleman the opportunity to visit the places inhabited by a large Italian community. The epistemological approach being adopted in the study, which is a geographical one, takes into consideration the historical and cultural context in which the author's political beliefs developed: his interpretation of the landscape and the places he describes mirrors his choice of sometimes favouring colonial propaganda "Occidental" over the narration of events.
\end{abstract}

Keywords: Tunis, Guglielmo Collotti, Italian colony in Tunisia, Medina, European headquarter, Italian College.

Riassunto. L'obiettivo del presente lavoro è quello di ricostruire il ritratto (geo)letterario della città di Tunisi, attraverso l'analisi dell'opera di Guglielmo Collotti intitolata Tunisi e il suo popolo. Studi impressioni e ricordi e pubblicata a Catania nel 1876. Si tratta del resoconto di un viaggio pianificato col pretesto di consegnare un premio "filantropico" al sovrano della Reggenza tunisina ma che, in realtà, ha permesso al nobile siciliano di visitare i luoghi e gli spazi in cui risiedeva la folta comunità di italiani. L'approccio epistemologico, in chiave geografica, utilizzato per questa indagine tiene in considerazione il contesto storico-culturale dellepoca in cui si inserisce il pensiero politico dell'autore: l'interpretazione del paesaggio o dei luoghi della narrazione diventa specchio riflesso di una volontà che, in alcuni casi, attenua il racconto dei fatti per privilegiare una propaganda riconducibile all'ideologia coloniale "Occidentale".

Parole chiave: Tunisi, Guglielmo Collotti, colonia italiana in Tunisia, Medina, quartiere europeo, Collegio italiano. 


\section{Introduzione}

Nell'estate del 1873, il catanese Guglielmo Collotti de' Baroni di San Pietro intraprendeva un viaggio "diplomatico" con lo scopo di consegnare un'onorificenza a Muhammad III as-Sadiq Bey di Tunisi. Il riconoscimento destinato al sovrano tunisino, consistente in una medaglia d'oro, veniva attribuito dalla Lega Giovanile Nazionale, istituzione filantropica istituita nel 1870 dallo stesso Collotti, che annualmente assegnava dei premi. La missione, durata solo poche settimane, divenne l'oggetto di un dettagliato resoconto di viaggio intitolato Tunisi e il suo popolo. Studi impressioni e ricordi, che il siciliano pubblicò nel 1876.

Quest'opera, sul piano testuale, costituisce un proficuo dialogo tra discorso letterario e rappresentazione geografica. I contenuti da un lato offrono un ampio affresco delle caratteristiche storiche, geografiche e sociali della Reggenza tunisina, dall'altro si distinguono per il richiamo a un certo patriottismo coloniale che guardava all'Africa come a una terra "primitiva", le cui popolazioni necessitavano di essere liberate dalle restrizioni imposte dalle società vigenti. Il processo narrativo imbastito da Collotti è strutturato attorno a una dicotomia in cui viene riconosciuto sia il lodevole impegno dell'attuale "sovrano della Tunisia, [che] coi suoi nobili sforzi, ha contribuito a migliorare la sorte di quei popoli barbari" (Collotti, 1876, p. VI) - probabilmente riferendosi alle riforme sulle libertà religiose, le uguaglianze civili e la carta costituzionale intraprese in quegli anni -, sia l'indispensabile "supporto" delle culture europee, per cui l'autore auspicava "che l'Italia abbia quindi la gloria di essere maestra di civiltà a quella fertile regione [...], tesoro sepolto nell'inerzia e nell'ignoranza" (Collotti 1883, 10-11).

All'interno di questa prospettiva, l'interpretazione del paesaggio diventa specchio riflesso di una visione che in alcuni casi attenua il racconto dei fatti per privilegiare una certa propaganda riconducibile all'ideologia coloniale (Brioni, Shimelis 2018); premesso ciò, le descrizioni della città ricavate dall'opera di Collotti nel complesso appaiono come un insieme di testimonianze che permettono di leggere e di definire, attraverso un'adeguata analisi delle culture urbane, le pratiche, le mutazioni, le evoluzioni degli spazi e delle identità (Borghi, Camuffo, 2010). In questo contesto "il testo letterario assume [...] un ruolo centrale nella ricerca geografica, non solo come fonte di informazioni o come espressione simbolica di esperienze territoriali, bensì anche come documento geografico a sé stante, oggetto centrale di ricerche volte a riflettere sul valore soggettivo della relazione tra individui e luoghi” (Gavinelli 2009). Ad oggi, la valenza delle opere letterarie come fonti per la conoscenza geografica è ampiamente riconosciuta (Persi, Dai Prà 2001; Lando 2005; Gavinelli 2007; Papotti 2011; Lucchesi 2012; Marengo 2016); tra queste, particolare attenzione è anche dedicata alla narrativa e alla produzione odeporica in cui si privilegia la prospettiva geografica dove il criterio spaziale è assunto come forma primaria d'indagine (Corna et al. 1987; Papotti 2003; Dai Prà 2019; Gabellieri 2019).

Le caratteristiche argomentative contenute in questo resoconto di viaggio sembrano corrispondere a quelle che Dino Gavinelli ha recentemente definito le "insidie" della produzione letteraria odeporica: circostanze capaci di produrre uno scollamento tra gli interessi del geografo e l'espressione dello scrittore, portando a percepire e a indagare in maniera differente il medesimo paesaggio o contesto ambientale. Si tratta di fonti narrative che, se utilizzate come strumenti di ricerca, necessitano dell'uso di una certa cautela nel decodificare descrizioni, simboli ed elementi carichi di valenze territoriali (2019).

Sulla base di queste considerazioni metodologiche, si cercherà di effettuare una ricostruzione geostorica degli spazi urbani e delle influenze ideologiche culturali che, in chiave socio-territoriale, hanno "condizionato" la trama dell'opera, attribuendo al linguaggio letterario l'abilità e la capacità di fissare nei luoghi e nei paesaggi i significati pensati (Dai Prà 2003; Maggioli, Morri 2010, 58; Guarducci, Rombai 2017; Nicosia 2020).

\section{Breve quadro biografico}

Guglielmo Collotti de' Baroni di San Pietro nacque a Catania il 7 febbraio 1851 (Tumino 2013) (Fig. 1). Da giovanissimo diresse L'Archimede, una rivista di pedagogia, didattica e questioni scolastico-giuridiche edita in Catania dal 1868 al 1872 (Collotti 1871). Nel 1871 era già laureato nelle lettere ${ }^{1}$.

Collotti, nell'ottica di un concreto e attivo impegno sul territorio, si fece promotore di un'associazione denominata Lega Giovanile Nazionale di mutuo soccorso e di incoraggiamento che egli stesso aveva istituito nella città etnea il $1^{\circ}$ settembre 1870 . Nel dicembre dello stesso anno, l'istituzione raccoglieva già sessanta soci di "prima classe" e tra questi vi erano numerosi esponenti ari-

\footnotetext{
${ }^{1}$ L'informazione viene riportata sul frontespizio del volumetto scritto dallo stesso Guglielmo Collotti, Religione e Progresso. Lettera - Risposta allamico Cavaliere Enrico Rodolfo de Angelis, del 1871. Si è cercato un riscontro della notizia presso l'Archivio Storico dell'Università degli Studi di Catania ma a causa di una significativa lacuna documentaria che riguarda proprio la Facoltà di Lettere negli anni fra il 1861 e il 1887, l'indagine non ha portato ad alcun esito.
} 


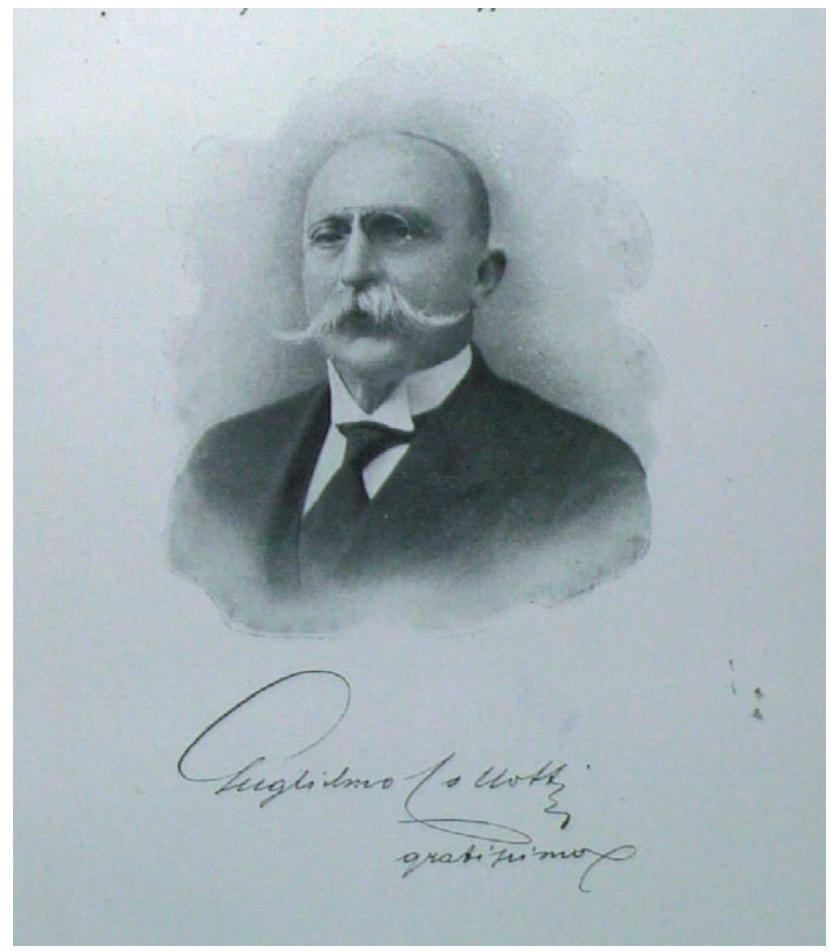

Figura 1. Guglielmo Collotti. Fonte: Collotti 1917.

stocratici locali, professori universitari e nomi noti della Catania dell'epoca (Collotti 1872, 5). Il sodalizio si prefiggeva di incoraggiare i giovani allo studio e di aiutare economicamente, attraverso appositi sussidi, gli studenti che "per essere privi di mezzi, siano impossibilitati a continuare il corso dei loro studi" (Collotti 1872, 24). "L'Archimede" fu quindi integrato come organo ufficiale della nuova istituzione e nel 1873 sia la rivista che la Lega furono assorbite da L'Educatore siciliano, periodico allora diretto dal professore catanese Sante Giuffrida.

Nel 1871 il Collotti, aveva già accumulato una lunga sfilza di riconoscimenti e una significativa quantità di affiliazioni a società e accademie italiane e straniere. Come altri uomini del suo tempo, assunse l'impegno civile come una vocazione in cui l'iniziativa morale si traduceva in uno strumento con cui alimentare il dibattito culturale. Nel suo Discorso programma pronunciato nel 1871, in maniera apertamente provocatoria, affermava che "Quando l'alfabeto si sostituirà alla polvere [da sparo], quando i milioni non più si semineranno negli arsenali né nelle caserme, ma si spenderanno nelle scuole che unicamente creano la civilizzazione dei popoli, mentre la guerra invece la uccide, allora questo popolo saprà rendersi meritevole della libertà più di noi, i quali non abbiamo né potevamo seguirla senza molti errori, per la semplice ragione di non esservi educati" (Collotti 1872, 51).
Nel 1873, nel ruolo di Presidente della Lega, intraprese un viaggio in Tunisia, allora al centro delle mire colonizzatrici dell'Italia, fermandosi ad annotare le geografie dei luoghi, gli usi e i costumi del popolo nordafricano, che tre anni dopo confluirono nel volume Tunisi e il suo popolo. Studi, impressioni e ricordi. Un'opera premiata e in diversi contesti anche molto apprezzata ${ }^{2}$ che, attraverso le pagine di alcuni quotidiani nazionali come quelle de Il Torino, proiettò le idee di Collotti al centro del dibattito in cui si criticava apertamente l'immobilismo diplomatico dei governi di Agostino Depretis e Benedetto Cairoli (Collotti 1883). Questa attività pubblicistica lo portò ad intraprendere uno scambio epistolare anche con Giuseppe Garibaldi, con il quale condivideva idee, preoccupazioni e speranze patriottiche (Ximenes $1885,256)$.

Dal 1877, già fervente patriota e ufficiale superiore dell'esercito, si dedicò all'insegnamento nelle scuole tecniche. Nel febbraio dello stesso anno iniziò la sua attività professionale presso la Regia Scuola Nautica di Pizzo Calabro come professore di Storia, geografia e diritto. Durante questa prima esperienza didattica maturarono le condizioni per la stesura dell'opera intitolata Sulle scuole primarie di Pizzo Calabro, edita a Catania nel 1877. L'incarico fu tenuto fino a quando rientrò a Catania dove, il $1^{\circ}$ maggio 1880 , fu nominato direttore della prima Scuola tecnica Agatino Sammartino (Tumino 2013), che era stata aperta nella città etnea nel 1861 (Fusto 2012). Contestualmente al ruolo dirigenziale, in questi anni presso la stessa Scuola tecnica fu professore incaricato di "Storia e geografia, diritti e doveri". Nel 1902 Collotti diede inizio alle stampe di una serie biografica dedicata ai Cavalieri del lavoro, attraverso la quale illustrava ai più giovani il valore educativo del lavoro e le virtù del sapere che insieme conducevano al miglioramento economico e morale ${ }^{3}$. Concetti da sempre espres-

${ }^{2}$ Nel 1878 il volume venne anche premiato con medaglia d'argento dall'accademia francese Confucius di Bordeaux (Collotti 1883, 17).

${ }^{3} \mathrm{Si}$ tratta di una collana composta da otto serie editate tra il 1902 e il 1904 a Catania presso l'editore Nicolò Giannotta. Le biografie furono raccolte in agili volumetti con destinazione educativa e sono stati firmati cono lo pseudonimo Cigo, corrispondente appunto a Collotti Guglielmo (Collotti, G. [Cigo] (1902), I cavalieri del lavoro. Serie I (F. Dorigo, P. Franci, N. Giannotta, E. Mele, A. Oldrini, A. Ravera, M. Sangiorgi, G. Spinelli fu Bruno, A. Tosi, A. Zago); id. (1902), Serie II (G. Appiani, L. Bonavita, C. Cacace, P. Camerini, F. Cavessago, F. De Blasio, G. Grigolon, C. Grimaldi, G. Gussoni, P. Milesi, P. Pontecorvo, F. Prina); id. (1903), Serie III (B. Camona, L. Carrera, R. Caruso, A. Centurini, C.B. Crespi, P. Di Bella, E. Faina, E. Mari, F.P. Materi, B. Mazza, P. Miliani, E. Ponti, E. Rizza, E. Strada); id. (1903), Serie IV (G. Ainis, P. Antoniotti, G. Beccaro, S. Biondo, V. Boero, A. Castellani, C. Gerli, E. Maraini, G. Penotti, G.B. Porazzi, d. Raffaelli, E. Rizzi, A. Rostain, G. Tempioni, L. Zotti); id. (1903), Serie V-VI (G.B. Buitoni, C. Castagna, E. Cravero, G. Di Rovasenda, V. Feo, F. Lombardi, A. Lucchini, A. Martini, E. Moriondo, G. Novi, 
si se si pensa che già nel luglio 1871, in occasione della distribuzione dei premi della Lega agli alunni nella sala comunale del municipio di Catania, agli studenti presenti rammentava che "Questo premio segna l'èra del vostro morale risorgimento-questo premio vi addimostra, come la penna non è il privilegio dei pochi; poiché le incallite mani dell'operajo, possono anche usarla, e forse con maggior coscienza d'ogni altro. Il ferro del vostro mestiere vi procurerà il vitto quotidiano; ma la penna vi alimenterà il cuore ai nobili principj che formano l'onesto cittadino, vi squarcerà il fitto velame della superstizione, in cui il governo dei despoti e l'arbitrio dei preti, vi aveano avvolto" (Collotti 1872, 37).

Negli ultimi anni della sua carriera professionale, conclusasi intorno al 1917 (Collotti 1917), partecipò attivamente ai lavori nazionali riguardanti la riforma della scuola, contribuendo al dibattito sullo svecchiamento dei ruoli e al miglioramento delle condizioni economiche/ professionali degli insegnanti. Collotti morì a Catania il 18 marzo 1923.

\section{Tunisi e il suo popolo: motivazioni e contesto storico}

Il volume Tunisi e il suo popolo. Studi, impressioni e ricordi fu pubblicato nel 1876 a Catania presso la Coco Editori. L'opera si articola in più sezioni; una lunga nota dedicata "al lettore" precede una narrazione dei principali accadimenti storici e delle diverse dominazioni che hanno interessato la regione tunisina; segue una dettagliata descrizione della città di Tunisi e delle aree circostanti; nelle parti successive l'attenzione dell'autore si concentra sul racconto degli "usi, costumi e credenze" del popolo, sul governo e sui diversi aspetti dell'apparato amministrativo locale e, infine, sui "prodotti, industria e commercio" delle zone visitate. Si tratta di un sapere in molti casi filtrato dall'uso di atlanti, di opere storicogeografiche dedicate alla Tunisia e di una più ampia letteratura di viaggio coeva; quest'ultima permette ai lettori di oggi di cogliere alcuni dei topoi letterari a cui l'autore fa ricorso per sostenere un'argomentazione.

Nel 1873, Collotti propose al comitato della Lega di assegnare il primo premio al Bey di Tunisi, ma la richiesta generò molte perplessità e non venne accolta con favore. A non essere condivisa era l'idea di legittimare con un'onorificenza "un monarca maomettano, signore

D. Orsini, G. Sarica, S. Solari, G. Sosso, E. Stucchi); id. (1904), Serie VIIVIII (F.P. Amato, a. Armanino, E. Carugati, F. De villagomez, G. Gioia, G. Marconi, A. Mendola, G. Nardi, G.L. Pecile, A. Pederzoli, E. Petrobelli, F. Piacenza, C. Pintacuda, G. Quartara, L. Salvadori e A. Zanetti). assoluto d'un trono, ove la civiltà non ha penetrato che a rilento" (Collotti 1876, V-VI). A sedare le divergenze e lo scetticismo iniziale dei soci fu l'intervento dello stesso presidente, il quale spiegava che l'operazione si inseriva all'interno di un disegno molto più ampio volto a dare visibilità all'istituzione stessa. A tal proposito li esortava a tenere presente come "L'Italia [...], che oramai può vantare quell'unità a cui si sacrificarono milioni di martiri, deve ora ad ogni costo spandere i suoi lumi di progresso sulla vicina costa della Barberia" (Collotti 1876, VIII).

I motivi che spingevano Collotti a seguire questa linea erano alimentati dalla speranza che "l'Italia vorrà $\mathrm{o}$ presto $\mathrm{o}$ tardi stendere attraverso il mare una sua mano a quella figlia dell'Africa, per sollevarla dalle sue sventure, per ritornare qual fu un tempo bella e potente" (Collotti 1876, VIII). Secondo l'autore uno degli approcci possibili a supporto dello scopo era quello geografico culturale: "A me pare, che pria di tutto si debba studiare la natura del suo popolo; conoscerlo e giudicarlo meglio, che finora non si è fatto" (Collotti 1876, VIII-IX).

Il contesto storico nazionale in cui va inserita l'opera era quello di una Italia da tempo interessata al controllo coloniale della Tunisia. Le ragioni che spingevano la politica del Regno a pensare alla colonizzazione della reggenza tunisina erano in buona parte motivate dalla presenza nell'area di una folta comunità di italiani che, agevolata dalla vicinanza alla penisola, aveva abitato il paese sin dall'inizio dell'Ottocento. Le attività italiane avevano contribuito alla creazione di una fitta rete di scambi commerciali, gettando le basi per il processo di modernizzazione e di sviluppo avviato dalla nazione tunisina nella seconda metà del XIX secolo (Pendola 2007). Una delle riforme più incisive, nel 1857, fu quella del Patto fondamentale con cui veniva riconosciuta la libertà religiosa e l'uguaglianza davanti alla legge per tutti gli abitanti del paese, indipendentemente dalla loro religione. Con un decreto del 30 agosto 1858 venne istituto il primo governo municipale moderno per la città di Tunisi. A queste azioni, nel 1861, seguì la promulgazione della prima costituzione nel mondo musulmano, che però venne sospesa nel 1864 a seguito di una rivolta anti-fiscale ${ }^{4}$.

All'interno della comunità italiana, soprattutto nella prima metà dell'Ottocento, avevano trovato rifugio anche numerosi lavoratori specializzati, intellettuali e attivisti politici provenienti dalla penisola, tra cui anche Giuseppe Garibaldi. Il processo di integrazione dei nuovi abitanti fu mediato da proprie istituzioni e organizzazioni, tra cui

\footnotetext{
${ }^{4}$ La riforma costituzionale fu voluta da una parte dei dignitari e sollecitata da due potenze europee (il Regno Unito e la Francia). Questa carta costituzionale fu mal compresa e soprattutto mal ricevuta dalle popolazioni del Beylik di Tunisi, all'epoca provincia dell'Impero ottomano (Khadhar 1989).
} 
scuole e ospedali, la camera di commercio, un orfanotrofio, la società Dante Alighieri e la stampa di giornali in lingua italiana, capaci di preservare e favorire la propria appartenenza linguistica e culturale (Michel 1941).

Tra il 1861 e il 1870 gli italiani che emigrarono nella reggenza nordafricana furono tantissimi. Nei dati del primo censimento degli italiani all'estero del 1871, quelli in Tunisia erano esattamente 5.889 , per poi arrivare a raddoppiare nel successivo rilevamento del 1881 (Francolini 1939, 266). Ad attraversare il Mediterraneo erano soprattutto quelle classi di lavoratori non qualificati vittime della crisi agraria, nella stragrande maggioranza dei casi provenienti dalle isole di Sicilia e Sardegna (Melfa 2008). Il vertiginoso aumento degli italiani in Tunisia verificatosi in quegli anni, rappresentava uno dei principali motivi per cui i due paesi siglarono l'8 settembre 1868 un Trattato di commercio. Il concordato stabiliva il principio della "nazione più favorita" con cui veniva privilegiata l'Italia, al fine di incrementare gli scambi economici e offrire alla comunità italiana, già residente nel paese nordafricano, maggiori garanzie sul diritto di possedere beni immobili e agevolazioni fiscali pari a quella dei cittadini tunisini (Caparelli 1939).

Ad amplificare le attenzioni e gli interessi italiani sulla Reggenza in quegli anni fu anche la missione esplorativa organizzata dalla Società Geografica Italiana, in cui fu vagliata l'audace possibilità di "creare" un mare interno nella parte del Sahara al confine tra Algeria e Tunisia, attraverso la realizzazione di un canale tra il mar Mediterraneo e il lago Melrhir ${ }^{5}$ (Cerreti 2000). Le circostanze erano ben note anche alla Francia che, già nel 1873, attraverso l'operato del capitano François Élie Roudaire, aveva effettuato alcuni rilevamenti sul livello dell'area di Melrhir (Margot 2003; Natili 2008).

A sostenere finanziariamente l'impresa italiana avviata nel maggio del $1875 \mathrm{fu}$ il barone Giacomo Castelnuovo, deputato del Regno e socio della Società Geografica Italiana, i cui interessi sul territorio tunisino erano numerosi, motivo per il quale aveva già contribuito alla realizzazione del trattato del 1868 (Antinori et al. 1875). I risultati della spedizione furono discussi durante il Congresso geografico tenutosi nel settembre del 1875 a Parigi e presentati in diversi contributi, soprattutto sulle pagine del Bollettino della Società Geografica Italiana (Brunialti 1876).

Un altro evento che contribuì a mantenere la questione tunisina al centro del dibattito nazionale fu la missione in terra d'Africa del 1878 voluta dal Presiden-

\footnotetext{
${ }^{5}$ Il Melrhir era una depressione chiusa la cui altitudine era al di sotto del livello del mare. La regione era situata nel deserto algerino a 250 chilometri a ovest del golfo tunisino di Gabes (Carazzi 1972)
}

te del Consiglio Cairoli e affidata all'onorevole Giovanni Mussi, uomo legato ai circoli economici finanziari italiani, soprattutto milanesi, che aveva già condotto una ricognizione nella reggenza nel marzo dello stesso anno su incarico di Depretis. Nelle sue considerazioni finali il diplomatico italiano avanzò proposte per un rafforzamento della posizione italiana nella regione; tra queste, per esempio, la fondazione di una colonia agricola per gli emigranti italiani (Brunialti 1897, 363-367).

Ad ambire alle stesse intenzioni coloniali dell'Italia era anche la Francia che sulla costa settentrionale dell'Africa aveva già avviato, dal 1830, una graduale occupazione della vicina Algeria (Manacorda, Nouschi 1964). L'azione di governo di Cairoli, come anche quella guidata da Depretis, non ritenne mai opportuno procedere a un'occupazione, essendo in generale ostile a una politica militarista (Calchi Novati 1990). Nel maggio 1881, mentre nel Regno ancora si discuteva dell'eventualità o meno di un'azione sull'altra sponda del Mediterraneo, il governo della terza repubblica francese di Jules Ferry, ben consapevole dei propositi coloniali del Regno d'Italia, inviò un contingente di duemila uomini a Biserta, stabilendo di fatto un protettorato militare sulla Tunisia. Questa azione fu definita dalle cronache giornalistiche italiane "lo schiaffo di Tunisi", un'espressione con la quale si voleva evidenziare l'umiliazione subita dalla diplomazia estera italiana.

La debolezza politica del Bey non poté fare nulla per impedire l'occupazione francese, che venne ufficialmente sancita con il trattato del Bardo (12 maggio 1881). Gli italiani continuarono comunque a rappresentare la colonia "straniera" più popolosa presente in Tunisia: basti pensare che nel 1901 erano presenti nella regione circa centomila italiani e solo 24 mila francesi. Per tali ragioni, il Paese del Nord Africa veniva di fatto ancora considerato "italiano".

\section{La città e i suoi dintorni}

Nel luglio 1873, accolto dal Commendatore dottore Luigi Pinna, Console di Sua Maestà il Re d'Italia in Tunisia, Collotti approdò a La Goletta, una località posta sulla costa nordafricana dinanzi Tunisi. Nel tragitto verso la capitale, lungo la strada che costeggiava il grande stagno, l'attenzione del viaggiatore siciliano fu subito catturata dalla fisionomia del capoluogo che ricordava quella "d'un ferraiuolo aperto e steso, che corrisponde quasi ad un trapezio" (Collotti 1876, 40). La città, con le sue case bianche generalmente ad un piano intervallate dai minareti delle moschee, si estendeva sul declivio delle colline che digradavano verso il lago, un'ampia distesa 
d'acqua che la separava dal mar Mediterraneo. All'ospite occidentale venne fatto notare come la superficie della città, che in questi decenni raccoglieva una popolazione di circa 175.000 abitanti (Nuova Enciclopedia 1848), cominciava ad essere "alquanto modificata per le recenti fabbriche, erette principalmente ad allargare il quartiere abitato dagli europei" (Collotti 1876, 40).

A catturare l'interesse di Collotti fu anche il paesaggio rurale attraversato. Le colline circostanti si mostravano discretamente coltivate, anche se a suo parere non sufficientemente sfruttate dagli agricoltori locali. Da queste si ricavavano principalmente frumento e orzo ma anche gran turco, avena e diverse tipologie di legumi. Dalle produzioni arboree si ottenevano inoltre "l'uva, i fichi, le ciriege, le susine, gli albicocchi, le melagranate e principalmente i cedri, i limoni e le melarance [...]. I fichi d'India, che si propagano rapidamente ovunque, sono ivi in grande abbondanza e servono di siepe impenetrabile ai giardini. Le viti si coltivano alte, a forma di pergolati ed il fusto s'incontra spesse volte, grosso quanto quello di un albero. Gli oliveti sono estesissimi e di una produzione maravigliosa. Le foglie di cotesti alberi si rincontrano in quei paesi di un verde più cupo. L'ulivo selvaggio vi cresce abbondante. L'olio, che è di ottima qualità, è uno dei principali e pregevoli prodotti di esportazione, quantunque i tunisini nulla abbiano innovato circa al modo di estrarlo" (Collotti 1876, 194).

Secondo quest'ultimo giudizio, poco sembrerebbe essere rimasto dei tentativi avviati a inizio secolo finalizzati all'importazione di nuovi strumenti con cui migliorare le tecniche di trasformazione delle materie prime. Difatti, già nel 1817, il sovrano Mahmoud Pacha Bey aveva affidato "all'inventore genovese Bonfiglio, [...] la fabbricazione di dieci molini a grano, dieci da olive, e tre torchi pure per olive, ciascuno convenuto al prezzo di pezzi-duri di Spagna mille"6.

Per dare maggiore profondità alle descrizioni di Collotti, soprattutto in mancanza di un corredo iconografico nell'opera, verrà affiancata al testo un'immagine storica della città e della sua campagna, capace di restituire un migliore dettaglio informativo. Osservando la rappresentazione zenitale del territorio, disegnata dall'italiano Romano Rocchi e stampata a Torino presso gli stabilimenti della Salussolia nei primi anni dell'occupazione francese della Tunisia (1881), appaiono chiare la sua forma urbis e la disposizione dei vasti spazi abitativi (Fig. 2). Nella parte centrale della città, quella più antica e circoscritta da una prima cortina muraria, vi era la

\footnotetext{
${ }^{6}$ Archives Nationales de Tunisie, Correspondance du Royaume de Tunis et du Royaume de Sardaigne, Correspondance de 1798 à 1828, Dos. 612, Doc. 10, f. 240r.
}

Medina, un quartiere all'interno del quale si trovavano i più importanti edifici del potere civile e religioso. Ai suoi lati, protetti da una seconda cinta di mura, sorgevano due ampi e popolosi borghi, il Rebat Bab-El-Souika e il Rebat Bab-Ed-Djezira. Nello spazio compreso tra la Medina e il lago vi era, invece, il quartiere nuovo abitato dagli europei. La fonte cartografica mette in evidenza anche lo stato delle strade, strette e tortuose nella parte antica, più larghe e ortogonali tra loro nel quartiere degli europei (Sebag 1998). Un ultimo aspetto raffigurato è quello della rete ferroviaria che abbracciava la città da ponente a occidente, un impianto di linee ferrate costruite a partire dall'agosto del 1872 con la linea Tunisi-La Goletta (Debernardi 1963).

\subsection{I quartieri "storici"}

La città in quegli anni era ancora circondata da un antico ed efficace sistema di cortine murarie, con diverse torri bastionate e porte. $\mathrm{Al}$ suo interno, scrive l'autore, la salubrità dell'aria "non è molto pura, ciò interviene per la natura del suolo e principalmente per le fogne, che ad ogni piè sospinto emanano gl'insalubri loro miasmi, di cui non si cura l'abituale sudicia pigrizia degli abitanti" (Collotti 1876, 41).

Visitando i quartieri periferici, Collotti rimase letteralmente esterrefatto dalle condizioni di vita della gente che li abitava non riuscendo a comprendere come questa potesse "vivere in mezzo alle tante immondizie ammonticchiate lungo le vie, marcite come in un letamaio, emananti un puzzo insoffribile" $(1876,41)$. Le abitazioni sembravano delle buche, costituite da ambienti posti sotto il "livello della via, incastrata nella roccia, umide, senza aria e senza luce, tanto basse da obbligare a chinarsi chi vi entra. Là, sopra un pugno di paglia rannicchiasi una famiglia di cinque o sei individui, insieme ad un buon numero di animali più o meno domestici ed in contatto a tutta l'immondizia che si accumula" (Collotti $1876,41)$.

Nel vedere queste immagini lo stesso Collotti raccontava che dovette "retrocedere dinanzi alle miasmatiche esalazioni di quei locali, rattristato dell'abbrutimento in cui vive questa misera classe di popolo, oziosa, stupida, sofferente" $(1876,41)$ (Fig. 3).

Le case del popolo della classe media, invece, erano generalmente costruite con "mattoni incotti e riarsi al sole" (Collotti 1876, 45) e si sviluppavano su un unico piano, a differenza di quelle dei benestanti che si componevano di due o di tre piani, oltre il terrazzo. Strutturalmente non erano dotate di finestre che si aprivano verso l'esterno o ne avevano "appena una, coperta 


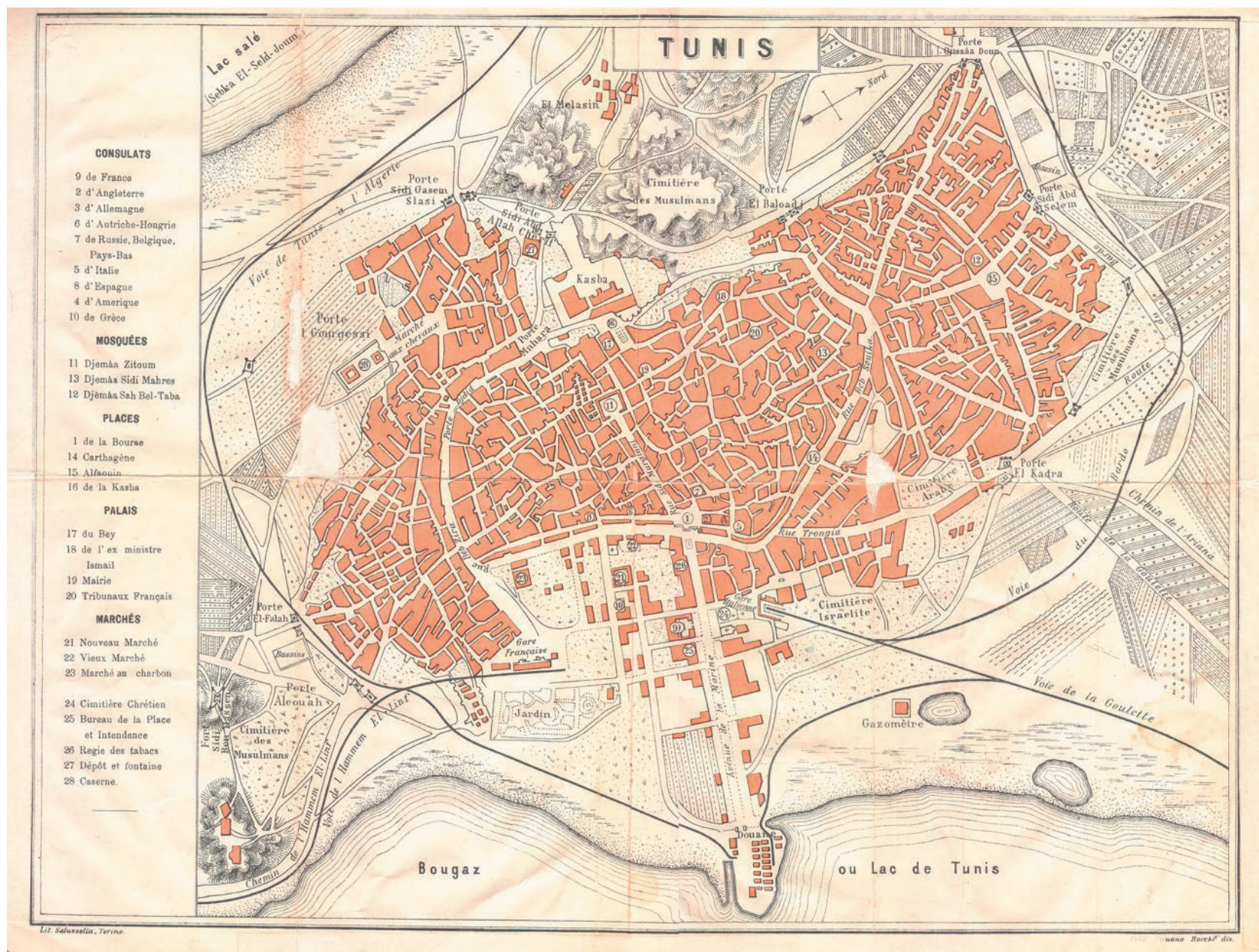

Figura 2. R. Rocchi, Tunis, Torino, s.d. [ma 1881-83 ca.]. Fonte: Bibliothèque Nationale de Tunisie.

da fitte grate, la gelosia degli abitanti non permettendo diversamente. V'è in alcune anche una specie di verone tutto rivestito di persiane, e non si apre che all'occasione di un Zeenak (grande solennità)" (Collotti 1876, 46). In queste costruzioni "La eleganza e la magnificenza architettonica, che tanto avevano in pregio gli antichi arabi, ivi si sconosce del tutto. Le fabbriche sono rozze e si presentano all'occhio con un incoerente miscuglio di ordini" (Collotti 1876, 45-46). Nella maggior parte dei casi, erano di forma quadrata e al loro interno vi era sempre un "atrio lastricato ordinariamente di marmo e cinto di colonne, pel quale si entra negli appartamenti, cui conducono varie porte disposte all'intorno. In un lato del cortile, avvi la finestra della cisterna, d'onde si attinge l'acqua pei bisogni della famiglia" (Collotti 1876, 46). Le strutture erano caratterizzate da una netta divisione dello spazio, espressione di una rigida separazione di funzioni e di compiti tra uomo e donna, che nelle abi- tazioni delle città del mondo musulmano costituiva storicamente una barriera che non mancava di manifestare i suoi simboli; da un lato si trovavano gli appartamenti degli uomini e dall'altro quelli delle donne, che abitavano le parti più interne della casa a causa di una evidente divisione del lavoro che le relegava ad una "clausura" e le rendeva invisibili agli occhi del visitatore (Aymard 1994, 132-133). Le stanze erano quasi sempre di forma rettangolare, strette e lunghe; i pavimenti, e spesso anche le pareti, erano ricoperti da mattoni verniciati.

I tetti delle abitazioni ubicate nella parte antica di Tunisi avevano le superfici piane, con una leggera inclinazione che permetteva la raccolta delle acque piovane nelle cisterne. Questi spazi comunicavano con l'interno della casa attraverso delle piccole scale di legno ed erano utilizzati anche come terrazze; in estate, nelle prime ore del tramonto, erano usate dalle donne come punto di ritrovo e per godere di un po' di refrigerio offerto dalle 


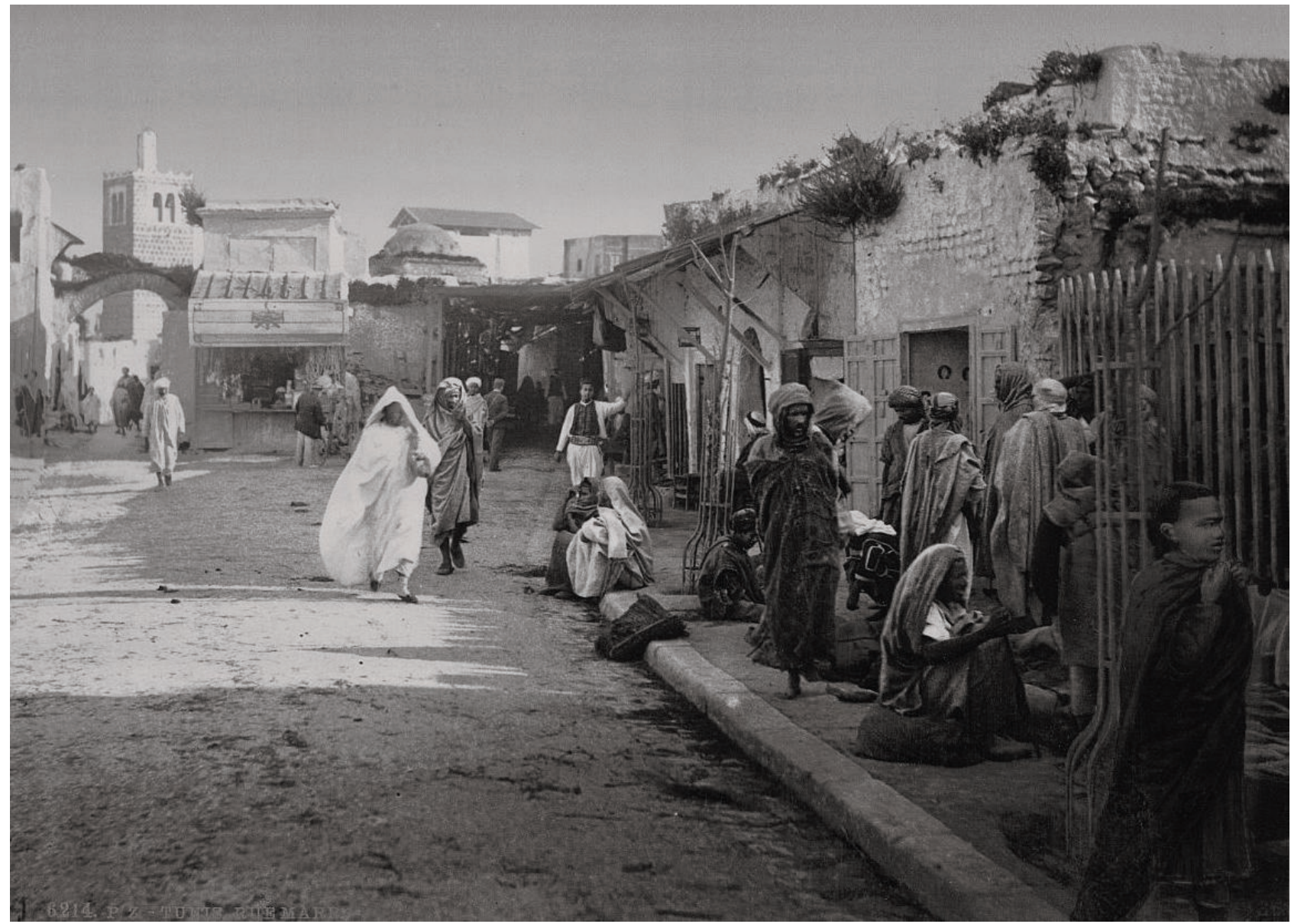

Figura 3. Rue du Marr, Rebat Bab-Ed-Djezira, Tunisi, (fine XIX secolo). Fonte: Library of Congress Prints and Photographs Division Washington (https://lccn.loc.gov/2001699382)

brezze del mare. Qui, venne raccontato a Collotti, frequentemente "si fanno le adunanze ed i festini, ove però non sono ammessi gli uomini, che assai di raro. La donna, vittima della gelosia moresca, in quel terrazzo scioglie un flebile canto d'amore e dà il racconto poetico delle sue sventure e delle sue sofferenze: spesso qual eco le risponde qualche galante trovatore, che piange l'amante lontana" $(1876,47)$. A Tunisi le tegole erano sconosciute (Fig. 4), infatti le terrazze erano coperte da un manto lastricato che, scrive il viaggiatore, "in Sicilia chiamasi battume ed è un cemento composto di due porzioni di cenere di legno, tre di calce ed una di sabbia, il tutto impastato con olio. Per renderlo consistente si batte per più giorni questo cemento, una volta disteso sulla parte che si vuol lastricare, la quale vien così coperta di uno strato duro e liscio come il marmo, impenetrabile all'acqua" $(1876,48)$.

I pavimenti delle case della classe media, a differenza di quelle dei ricchi che potevano essere lastricati di marmo, erano generalmente coperti con delle stuoie, perché per i tunisini era usanza non indossare le scarpe all'interno dei loro appartamenti.

Le caratteristiche architettoniche complessive, scrive Collotti, erano "l'emblema dell'attuale ignoranza di quei popoli, tanto ne è barocca la disposizione degli appartamenti. Dal cortile per salire al piano superiore, si sviluppa in fondo una scala ripidissima, sproporzionata" $(1876,46)$.

Le strade su cui si affacciavano gli edifici erano solitamente poco larghe e tortuose, in alcuni punti si restringevano così tanto da non lasciare passare nemmeno un uomo a cavallo. Si trattava di una conformazione a "camminamenti" volutamente scelta dagli stessi sovrani al fine di favorire la difesa dello spazio urbano in caso di sommosse o assedi; nei vicoli "quattro uomini soltanto possono fermare e combattere interi reggimenti. Per la stessa ragione molte fra le migliori case sono munite di feritoie, tanto da sembrare fortezze invece che abitazioni" (Collotti 1876, 42). Oltre alla loro architettura, un 


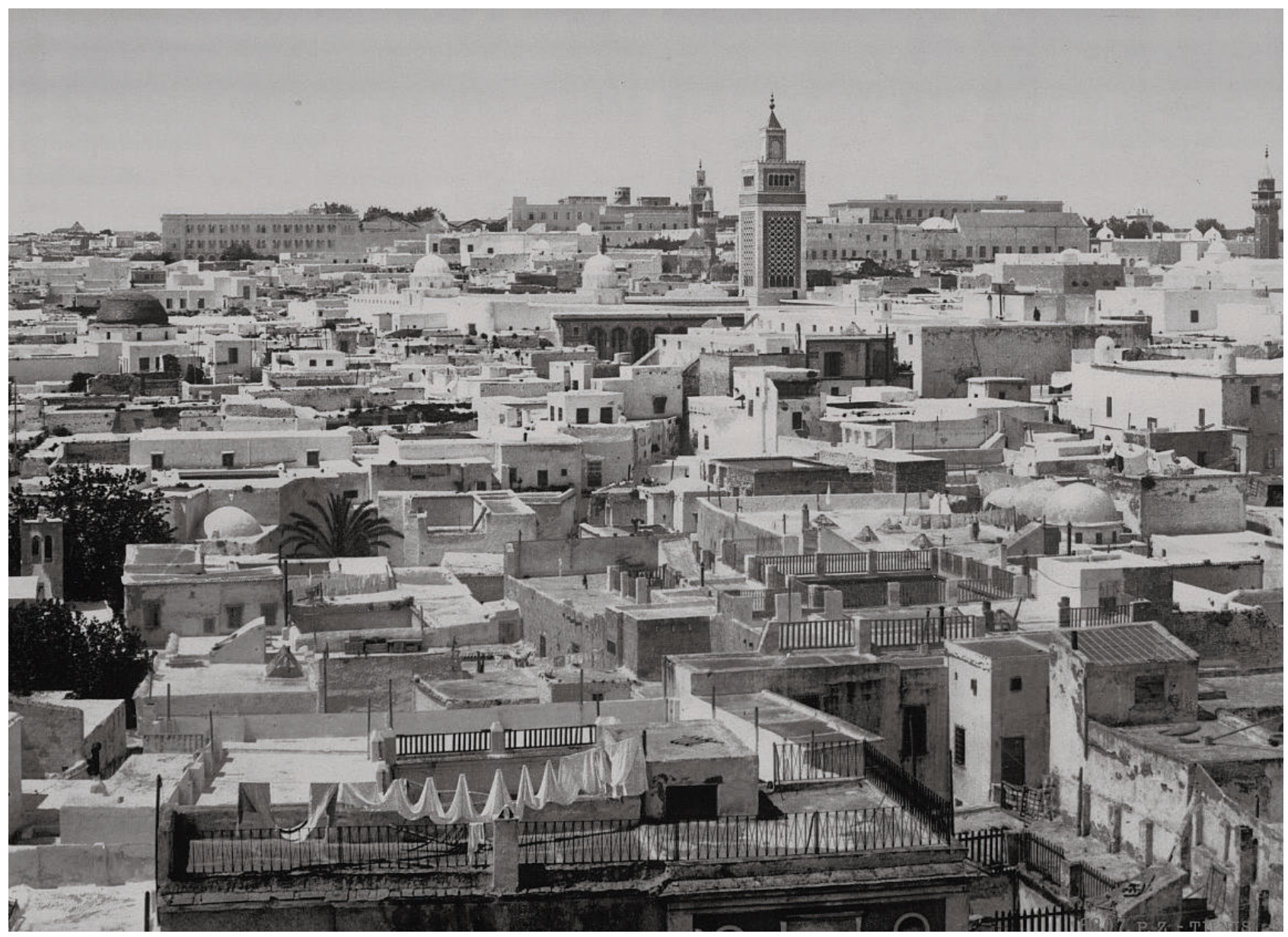

Figura 4. Vista della Medina di Tunisi, Tunisi, (fine XIX secolo). Fonte: Library of Congress Prints and Photographs Division Washington (https://lccn.loc.gov/2001699381)

ulteriore aspetto che caratterizzava questi luoghi era la scarsa sicurezza, "Per un europeo, nuovo nel paese, non è ben fatto avventurarsi solo in quell'intrigatissimo laberinto, perché in quei chiassuoli può facilmente trovarsi in non lieta compagnia" (Collotti 1876, 42). In queste stradine, purtroppo, si venivano a creare le condizioni ideali per i furti, soprattutto a discapito degli stranieri che diventavano facili prede di raggiri o malfattori. Succedeva spessissimo, infatti, che "quando l'arabo astuto ha visto qualche cosa di valore sulla persona di un forestiere, gli viene incontro di fretta, l'urta violentemente e fingendo avvolgersi nel suo vasto mantello, glielo caccia con tutta forza sul viso. Quando il mal capitato, riavutosi dal suo stupore, riapre gli occhi, il furto è consumato, il ladro è sparito in mezzo alla folla colla sua preda ed è impossibile rintracciarlo" (Collotti 1876, 44).

Nelle strade, anche quelle più trafficate, spesso "la pulitezza [...] è trascuratissima, sicchè la notte, le vie essendo totalmente al buio, si corre pericolo di affogare in qualche pozzanghera, molto più, che non poche chiaviche sono scoverte" (Collotti 1876, 43).

Tra i quartieri della città antica di Tunisi vi erano numerose piazze pubbliche. Fra queste, oltre a quella delle carceri e del castello, una delle più note e ampie era quella della Borsa, sulla quale, nella seconda metà dell'Ottocento, si affacciavano i palazzi consolari dei maggiori paesi d'Europa; lo slargo segnava anche il passaggio tra la Medina e il quartiere degli europei.

\subsection{I nuovi spazi urbani: il quartiere europeo}

La zona della città destinata ad accogliere "gli europei" era quella posta tra lo stagno e la Medina. Le case si trovavano ad un livello altimetrico più basso rispetto a quello in cui sorgevano i quartieri più antichi, che erano ubicati sul declivio della collina. Soprattutto nel 


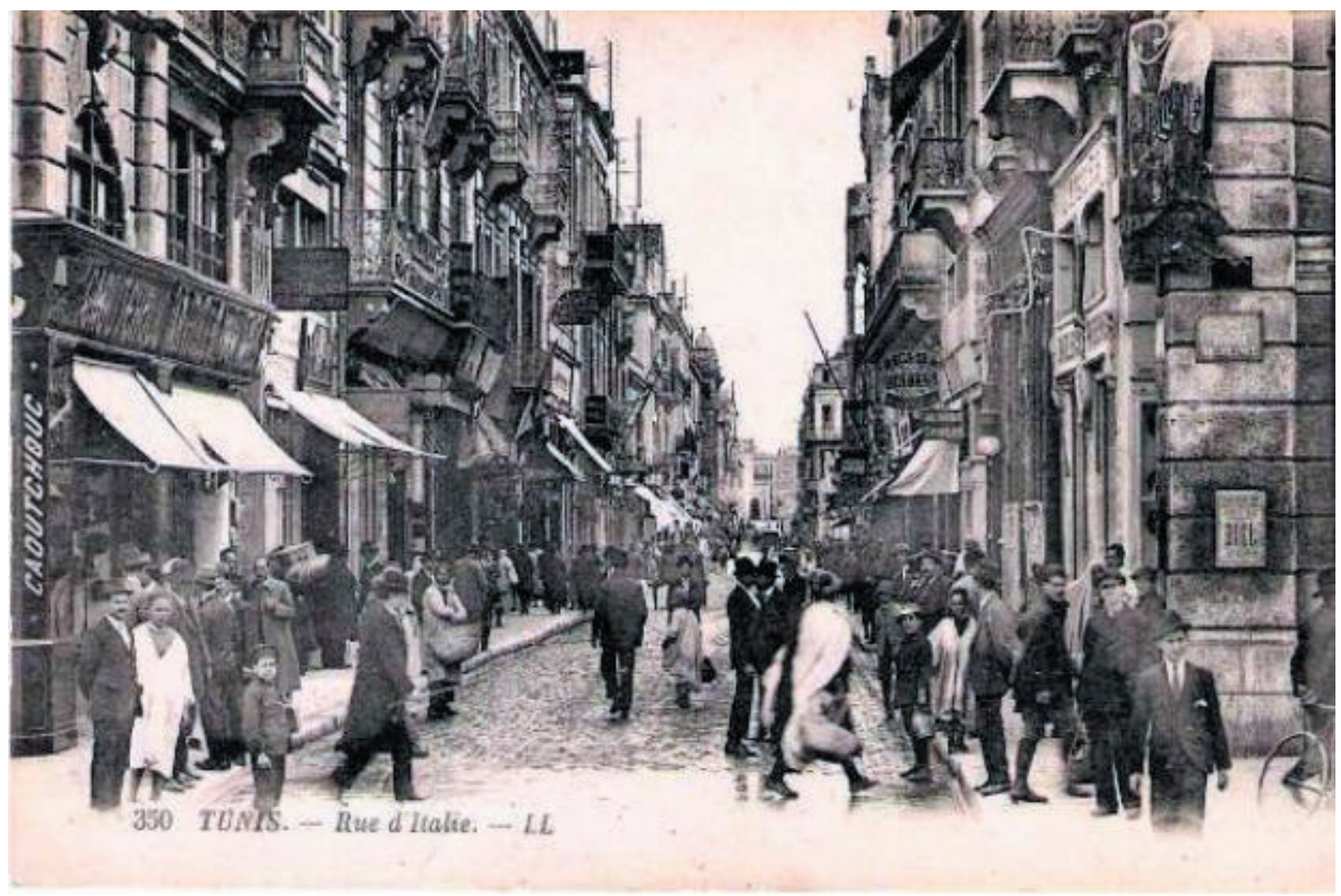

Figura 5. Rue d'Italie, Tunisi, (fine XIX secolo). Fonte: Library of Congress Prints and Photographs Division Washington (https://lccn.loc. gov/2001699394)

periodo invernale, una parte del nuovo quartiere diventava la meno salubre della città perché, come spiega Collotti, qui confluivano "Le acque piovane che si scaricano nel lago, trasportando tutte le immondizie della città, e cambiano in vera palude qualche parte di esso verso la sponda, sicché l'aria che vi si respira, non è molto sana; però a ciò si rimedia con la larghezza delle vie e costruendo le abitazioni alte ed ariate" $(1876,49)$. Difatti, le vie ortogonali tra loro che separavano gli isolati di case erano alquanto spaziose, ben tenute e per questo facilitavano gli interventi di pulizia (Fig. 5).

La strada principale, quella su cui convergevano la maggior parte delle vie secondarie, era l'asse viario che collegava la piazza della Borsa, dove si trovava l'antica Bab al-Bahr (porta del mare), allo slargo della marina in cui approdavano le imbarcazioni provenienti dalla Goletta. Il tratto finale di questa strada, quello più prossimo al lago e quindi alle case degli europei, era deputato alla passeggiata, uno spazio che l'estro occidentale aveva impreziosito con giardini e altre strutture per renderlo più ospitale e armonioso. Secondo Col- lotti, questa passeggiata era per Tunisi "Ciò che Chiaia è per Napoli, il Lungh'Arno per Firenze, la Marina per Palermo" $(1876,54)$. Si trattava di una pratica che, soprattutto nell'Ottocento, in Italia e in Europa era diventata un evento borghese, un vero e proprio fenomeno collettivo di natura sociale (De Ponti 2007). A Tunisi, nel 1873, l'area in cui aveva luogo la camminata era convenzionalmente riconosciuta e circoscritta geograficamente. Gli europei partecipavano alla passeggiata a piedi o in carrozza; aggirarsi in questi spazi era possibile fino alle ore dieci della sera, annunciate col suono della trombetta del governatore (Collotti 1876 , 55). In questo spazio, spiega Collotti, "girano nelle prime ore della sera gruppi di gentili donzelle italiane, inglesi e tedesche - allegre, vispe, saltellanti poco ossequenti alle prescrizioni della moda ed ai suoi capricci. Vi s'incontrano poi molte carrozze, che non hanno l'eleganza ed il gusto di quelle che percorrono le vie delle nostre città e sono guidate da arabi o mori, nel loro vestito uniforme, per nulla paragonabili ai nostri cocchieri in livrea" $(1876,54)$. 
Secondo le descrizioni di Collotti, lo spazio in cui si praticava la passeggiata era un luogo ormai convenzionalmente riconosciuto, in un contesto geograficamente ben circoscritto. Si trattava a quanto pare di un tentativo riuscito di ampliamento delle funzioni urbane della parte di città europea.

La prospettiva geografica a cui si rifà l'autore richiama quei tratti di strade urbane, piazze e viali che nella maggior parte dei Paesi occidentali erano comunemente destinati a questa abitudine sociale. Una caratteristica del paesaggio che, grazie all'incontro fra geografia e letteratura, è capace di alimentare inedite rappresentazioni narrative (Papotti 2003). Rintracciare le linee essenziali dell'interpretazione e dell'approccio non è semplice, ma gli scorci descritti e i modelli presenti in questa zona della città sembrano rimandare all'identità e ai luoghi di provenienza del viaggiatore (Nicosia 2020, 347-348).

\subsection{Un avamposto culturale: il Collegio italiano}

Gli individui che a metà Ottocento costituivano la collettività "italiana" nella Reggenza di Tunisi provenivano da tutti gli Stati della penisola ma per lo più dall'Italia meridionale e dalle isole. La lingua utilizzata era legata alla propria origine geografica e sociale (Pendola 2007, 76). A partire dal 1861 il Regno d'Italia diventò la madre patria dei diversi gruppi della colonia, riunendoli tutti sotto l'egida di un'unica grande comunità.

All'indomani dell'Unità i nuovi governi non fecero mancare alla colonia di connazionali l'appoggio per la creazione di una scuola maschile. I corsi furono inaugurati nel gennaio del 1864 e, poco meno di un decennio più tardi, raccolti sotto un'unica istituzione che prese il nome di Collegio italiano, al quale il nuovo Regno elargiva una sovvenzione economica annua per il mantenimento (Loth 1905, 376-377); sempre in questo periodo, per consentire agli allievi di proseguire gli studi, venne creata anche una Scuola tecnica.

Attraverso le preziose informazioni che l'autore dichiara di aver ricevuto grazie "alla gentilezza di un mio egregio amico", egli inserisce all'interno del suo resoconto di viaggio un ragguaglio completo e minuzioso dell'impegno che il nuovo Regno riservò all'istruzione degli italiani della colonia. Le motivazioni di tanto scrupolo sono da ricondurre direttamente ai suoi interessi professionali e all'attività intellettuale. Ci si trova di fronte a due argomenti, l'insegnamento da un lato e il manifesto impegno politico italiano verso la Tunisia dall'altro, su cui gravitavano, soprattutto in quegli anni, una buona parte degli impegni civili e politici esercitati del giovane siciliano.

Non si tralasci che Collotti già nel 1871 era presidente di un'istituzione morale che aveva lo scopo di favori- re la scolarizzazione delle classi meno agiate; pochi anni dopo il suo rientro in Italia, avrebbe iniziato la sua carriera di professore in Storia, geografia a diritto; inoltre, era a favore di una colonizzazione italiana della Tunisia.

Inizialmente, scrive il siciliano, il sussidio elargito dal governo italiano era di lire 3.500. Le classi avviate nel 1864 accoglievano "un totale di 73 allievi e 4 insegnanti, di cui uno funzionava qual direttore; l'istruzione non si estendeva oltre i programmi delle classi elementari. Il numero crescente di allievi indusse il Comitato amministrativo a far costruire l'attuale Collegio; a tal uopo il Bey regalò il terreno e la colonia per mezzo di sottoscrizioni e di un prestito sopperì alle spese, che ascesero alla somma di L. 50.000" (Collotti 1876, 50).

Nel 1865 veniva inoltre introdotta una classe di perfezionamento dedicata allo studio "della lingua francese: nel $1868 \mathrm{fu}$ istituita un'altra classe e nel 1870 si diede alfine ordine e programma all'attuale corso tecnicocommerciale, diviso in tre anni. Gl'insegnanti furono pure aumentati secondo il bisogno e man mano che si aprivano nuove classi: attualmente il corpo insegnante consta di nove persone, compreso il Direttore, cioè: quattro maestri elementari, un professore di francese, uno d'arabo, uno di disegno e due per le altre materie nelle classi tecniche. Il governo però andò accrescendo il sussidio, portandolo prima a lire 5.000 e da tre anni a 6.500" (Collotti 1876, 50).

Le attività didattiche del collegio erano mantenute dal sussidio governativo e dalle tasse pagate dagli studenti. Queste non erano uguali per tutti, erano mensili e variavano a seconda della classe che si frequentava. Erano pure previste delle forme di sostegno economico per gli iscritti. Ai corsi venivano ammessi "a mezza tassa gli allievi di famiglie italiane non agiate e gratis gl'italiani privi affatto di beni di fortuna. Gli allievi gratuiti sono in media $3 / 7$ del numero totale. Le tasse scolastiche, secondo la media degli ultimi tre anni, produssero un'entrata di circa L. 12.000, che unite al sussidio governativo, formano un totale di L. 18.500 di entrata. Le spese pareggiano quasi le entrate senza poter soddisfare molti bisogni, che ancor si notano per questo istituto" (Collotti 1876, 51). L'amministrazione e la gestione dell'istituzione era affidata ad una deputazione composta da sei notabili appartenenti alla colonia italiana e presieduta dal Console Generale che svolgeva anche le funzioni di dirigente scolastico.

A partire dal $1870 \mathrm{fu}$ pure avviata una scuola femminile che nel giro di poco tempo raccolse un buon numero di studentesse. Nel periodo in cui Collotti visitò la città, l'istituto era costituito da "quattro classi con programmi conformi alle scuole primarie d'Italia, ma si aggiunge alla classe $4^{\text {a }}$ lo studio del francese. Vi si danno pure lezioni 
di musica alle allieve che le pagano partitamente. La tassa delle allieve a pagamento è di Fr. 6 mensili, però circa la mettà imparano gratuitamente e sommano in totale ad una media di 160. Il corpo insegnante si compone di una Direttrice, due maestre e quattro sotto-maestre, oltre l'incaricato per il francese. Il sussidio annuo governativo è di Fr. 5000 - le entrate pareggiano le spese" $(1876,52)$.

L'offerta didattica, a supporto dell'istruzione e della cultura degli italiani della Reggenza, fu ampliata ulteriormente con l'istituzione di una sede succursale maschile del Collegio di Tunisi alla Goletta. La scuola nel 1873 era "frequentata da una sessantina di allievi e diretta da un maestro e da un sotto-maestro, che dipendono dal Direttore del collegio di Tunisi - Ha Fr. 2300 di sussidio governativo e poche altre fonti di entrata" (Collotti 1876, 52).

\section{Conclusioni}

Le strategie discorsive utilizzate da Collotti per la descrizione degli spazi urbani mettono in evidenza gli aspetti della formazione dell'identità del colonizzatore e del colonizzato (Coglitore 2020). Si tratta di un insieme di immagini narrative di un territorio che posto all'interno di una più ampia prospettiva geografica, offre preziose indicazioni con cui ricostruire le realtà paesaggistiche del passato (Papotti 2003, 393-394). Queste riflessioni e incontri interdisciplinari riportano al centro del dibattito il problema epistemologico della rappresentazione, interpretazione e concezione geografica con cui vengono definite le forme di interazione concepite e poi storicamente realizzatesi tra le parti (Bellagamba 2004, 98). Si tratta di considerazioni che non differiscono molto da quelle già espresse da Edward Said nei saggi Orientalismo (1991) e Cultura e Imperialismo (1998), dove viene delineato, offrendo una rinnovata analisi dell'argomento, il ruolo determinante e tutt'altro che innocente svolto dalla cultura e dalla letteratura occidentale nella legittimazione dell'esercizio del potere a discapito, in particolare, dell'Oriente. Si tratta di una produzione culturale che, attraverso tecniche ideologiche, propaganda, creazione di concetti e stereotipi, ha stigmatizzano le popolazioni arabo-islamiche e la loro cultura, rendendole differenti e incapaci di progredire autonomamente e "giustificandone" la loro "soppressione". Più genericamente, Said contesta il modo in cui l'Occidente rappresenta l'Oriente, una raffigurazione fortemente viziata da pregiudizi etnografici di varia natura, che porta inevitabilmente gli studiosi (spesso anche contro la loro volontà) ad operare seguendo categorie errate e quindi, ovviamente, a giungere a conclusioni altrettanto erronee.
Nel contesto qui indagato, la città, in particolare, è un dispositivo estremamente efficace per rivelare queste dinamiche. La concretizzazione narrativa urbana mostra una netta separazione tra gli spazi geo-letterari del "quartiere europeo" e quelli della Medina con i suoi borghi. La città antica permetteva alla popolazione locale di conservare le proprie abitudini e i propri stili di vita. Al contrario quella europea, costruita al di fuori delle mura, presentava i segni delle regole moderne della geografia urbana, di cui vengono messi in risalto la monumentalità della piazza, le strade larghe, la pulizia e il giardino pubblico con la sua passeggiata.

Appare chiaro che, nella trama argomentativa dell'opera di Collotti, la città non viene letta come spazio autonomo, inserito all'interno di un contesto, ma viene "costruita" attraverso il continuo confronto con il modello europeo, sinonimo in questo caso di civilizzazione. È lo stesso autore a spiegare che "senza il valevole appoggio di una nazione costruita e potente, la Tunisia non potrà partecipare al progresso dell'Europa. I raggi dell'astro che illumina attualmente il mondo civile giungono tanto pallidi attraverso il mare e i monti, da non poter per nulla diradare le tenebre, che ravvolgono quei popoli. Bisogna che le nazioni concedano un po' della loro luce ad una sorella, che affatto ne manca" (Collotti 1876, IX). L'opera, se da un lato mette in evidenza come il giovane componente dell'élite siciliana rappresenti al meglio l'originale versatilità geografica umanistica (Lando 2012; Lopez 2019) e politica di un uomo di cultura del suo tempo, dall'altro, in pieno spirito patriottico, esorta l'uscita dell'Italia dall'indecisa fase di puro raccoglimento di quegli anni e auspica l'inizio di un processo di espansione realizzabile mediante un'opera "civilizzatrice" di colonizzazione diretta.

\section{Ringraziamenti}

L'autore desidera ringraziare il dott. Salvatore Consoli, responsabile scientifico dell'Archivio Storico dell'Università degli Studi di Catania, per l'aiuto e la disponibilità offerta nelle ricerche; gli anonimi revisori che attraverso le importanti sollecitazioni di riflessione fornite hanno consentito di migliorare notevolmente il presente lavoro.

\section{Riferimenti bibliografici}

Aymard, M. (1994). Spazi. In Braudel, F. (a cura di). Il Mediterraneo. Gli spazi, la storia, gli uomini, le tradizioni. Milano, Bompiani, 123-144. 
Antinori, O., Vanzetti, A., Lambert, A., Bellucci, G. (1875). Spedizione italiana nella reggenza di Tunisi. Prima relazione presentata alla presidenza della Società geografica italiana. Bollettino della Società Geografica Italiana, 9(12), 453-468.

Bellagamba, A. (2004). Identità e storia nell'archivio coloniale del Gambia. In Casti, E., Corona, M. (a cura di). Luoghi e identità. Geografie e letterature a confronto. Bergamo, Edizioni Sestante, 93-118.

Borghi, R., Camuffo, M. (2010). Differencity: postcolonialismo e costruzione delle identità urbane. In Barberi, P. (a cura), È successo qualcosa alla città. Manuale di antropologia urbana. Roma, Donzelli, 117-149.

Brioni, S., Gulema, S. (eds.). (2018). The Horn of Africa and Italy: Colonial, Postcolonial and Transnational Cultural Encounters. Oxford, Peter Lang.

Brunialti, A. (1876). Il mare saharico e la spedizione italiana in Tunisia del Dottor A. Brunialti 1875. In Tunisi. Viaggi di Crapelet, Rebatel e Tirant.... Milano, Editori fratelli Traves, 193-213.

Brunialti, A. (1897). Le colonie degli italiani. Torino, Unione tipografico-editrice.

Calchi Novati, G. (1990). Cairoli, la Sinistra Storica e gli inizi della penetrazione coloniale in Africa: un caso di colonialismo controllato. Africa, 3, 445-464.

Caparelli, F. (1939). Civiltà italiana in Tunisia. Roma, S. A. Tipografia Editrice Italia.

Carazzi, M. (1972). La Società geografica italiana e l'esplorazione in Africa (1867-1900). Firenze, La Nuova Italia.

Cerreti, C. (2000). Della Società geografica italiana e della sua vicenda storica. Roma, SGI.

Coglitore, M. (2020). Viaggi coloniali. Politica, letteratura e tecnologia in movimento tra Ottocento e Novecento. Padova, Il poligrafo.

Collotti, G. (1871). Religione e Progresso. Lettera - Risposta allamico Cavaliere Enrico Rodolfo de Angelis. Catania, Tipografia Roma.

Collotti, G. (1872). Gli Atti della Lega Giovanile Nazionale del suo Primo Anno Accademico 1870-71, raccolti dal Presidente Cav. Gugl. Collotti dei Baroni di S. Pietro, dedicati alle Accademie e Società a cui egli appartiene con l'aggiunta di alcune sue pubblicazioni. Catania, Tipografia Galatola.

Collotti, G. (1876). Tunisi e il suo popolo. Studi, impressioni e ricordi. Catania, Coco Editore.

Collotti, G. (1877). Sulle scuole primarie di Pizzo Calabro. Catania, Tipografia popolare di Andrea Cavallaro.
Collotti, G. (1883). L'Italia in Africa. Lettere al giornale quotidiano Il Torino. Con due autografi di G. Garibaldi, uno de' quali in facsimile. Catania, Concetto Battiato Libraio-editore.

Collotti, G. (1917). Ricordando. Discorso pronunziato pel giubileo del $40^{\circ}$ anno del suo insegnamento. Catania, Giannotta Editore.

Corna Pellegrini, G., Scaramellini, G., Viola, G.E. (1987). I viaggiatori del Grand Tour in Italia. Milano, Touring Club Italiano.

Dai Prà, E. (2003). I parchi letterari italiani tra riproduzione e innovazione. Geotema, 20, 10-16.

Dai Prà, E. (2019). Il Viaggio in Italia di Goethe: ontologia del paesaggio nel solco della tradizione speculativa geografica (e non solo) tedesca? In Salvatori, F. (a cura di). Atti del $32^{\circ}$ Congresso Geografico Italiano (7-10 giugno 2017), Lapporto della Geografia tra rivoluzioni e riforme. Roma, A.Ge.I., 617-620.

De Ponti, P. (2007). Geografia e letteratura. Letture complementari del territorio e della vita sociale. Milano, Unicopli.

Fonnesu, I., Rombai, L. (2012). Toscana. Geografia e letteratura paesaggi di ieri e di oggi. Arezzo, Helicon.

Francolini, B. (1939). La Tunisia e il lavoro italiano, Bollettino della Società geografica italiana, serie 7, 4, 255-272.

Fusto, D. (2012). Le scuole a Catania nel XIX secolo. Tesi di dottorato di ricerca, Dottorato di ricerca in Storia della cultura, della società e del territorio in età moderna, Università degli Studi di Catania.

Gabellieri, N. (2019). Geografia letteraria dei paesaggi marginali. La Toscana rurale in Carlo Cassola. Firenze, All'Insegna del Giglio.

Gavinelli, D. (2007). Geografia e Letteratura. In Casari, M., Gavinelli, D. (a cura di). La letteratura contemporanea nella didattica della geografia e della storia. Milano, CUEM, 5-14.

Gavinelli, D. (2009, 17 ottobre), La didattica della geografia: contenuti interculturali, aspetti interdisciplinari e nuove forme di cittadinanza, presentazione al Seminario regionale della rete lombarda ELLIS. https://www.storieinrete.org/storie_wp/?p=899 [20/12/2020].

Gavinelli, D. (2019). Introduzione a Geografia e letteratura. Luoghi, scritture, paesaggi reali e immaginari. In Salvatori, F. (a cura di). Atti del $32^{\circ}$ Congresso Geografico Italiano (7-10 giugno 2017), L'apporto della Geografia tra rivoluzioni e riforme. Roma, A.Ge.I., 597-604. 
Guarducci, A., Rombai, L. (2017). Paesaggio e territorio, il possibile contributo della geografia. Concetti e metodi. Scienze del territorio, 5, 19-25.

Khadhar, H. (1989), La Révolution française, le Pacte fondamental et la première Constitution tunisienne de 1861 . Revue du monde musulman et de la Méditerranée, 52/53, 132-137.

Lando, F. (1999). Luogo territorio e paesaggio. I segni del radicamento: geografia e letteratura. In Cusimano, G. (a cura di), La costruzione del paesaggio siciliano: geografi e scrittori a confronto. Palermo, Annali della Facoltà di Lettere e Filosofia dell'Università di Palermo, 203-216.

Lando, F. (2005). Geografia e letteratura. Le modalità per un'interazione. In Lando, F., Voltolina, A. (a cura di), Atlante dei luoghi. Ipotesi per una didattica della geografia. Venezia, Libreria Editrice Cafoscarina, 13-32.

Lando, F. (2012). La geografia umanista: un'interpretazione, Rivista Geografica Italiana, 119 (3), 259-289.

Lopez, L. (2019). A geo-literary analysis through human senses. Towards a sensuous Camino geography. Emotion, Space and Society, 30, 9-19.

Loth, G. (1905). Le peuplement italien en Tunisie et en Algerie. Paris, Librairie Armand Colin.

Lucchesi, F. (2012). Sviluppi teorici e tematiche di indagine negli studi di Geografia umanistica: i paesaggi letterari e quelli cinematografici. ACME - Annali della Facoltà di Lettere e Filosofia dell'Università degli Studi di Milano, 65 (2), 193-220.

Maggioli, M., Morri, R. (2009). Tra geografia e letteratura: realtà, finzione e territorio. Quaderni del Novecento, 9, 53-70.

Manacorda B., Nouschi A. (1964). Gli Europei in Algeria dal 1830 al 1954. Studi Storici, 5 (4), 675-690.

Marengo, M. (2016). Geografia e letteratura. Piccolo manuale d'uso. Bologna, Pàtron.

Margot, L. (2003). Une mer au Sahara: mirages de la colonisation, Algerie et Tunisie, 1869-1887. Paris, Éd. Dé la Différence.

Melfa, D. (2008). Migrando a sud. Coloni italiani in Tunisia (1881-1939). Roma, Aracne.

Michel, E. (1941). Esuli italiani in Tunisia: 1815-1861. Milano, Istituto per gli studi di politica internazionale.

Natili, D. (2008). Un programma coloniale. La Società Geografica Italiana e l'origine dell'espansione in Etiopia (1867-1884). Roma, Gangemi.
Nicosia, E. (2020). Urbanscapes e memoria dei luoghi: un ritratto (geo)letterario di Catania. Il capitale culturale, 22, 337-367. DOI: 10.13138/2039-2362/2396

Nuova Enciclopedia popolare, ovvero Dizionario Generale di Scienza, Lettere, Arti, Storia Geografia... (1848). Tomo XII. Torino, Pompa Editori.

Papotti, D. (2003). Attività odeporica ed impulso scrittorio: la prospettiva geografica sulla relazione di viaggio. Annali d'Italianistica, 21, 393-407.

Papotti, D. (2011). Geografia e letteratura: affinità elettive e accoppiamenti giudiziosi. In Giorda, C., Puttilli, M. (a cura di). Educare al territorio, educare il territorio. Geografia per la formazione. Roma, Carocci, 248-257.

Pendola, M. (2007). Gli italiani di Tunisia. Storia di una comunità (XIX-XX secolo). Foligno, Editoriale Umbra.

Persi, P., Dai Prà, E. (2001). "Laiuola che ci fa...” Una geografia per $i$ parchi letterari. Urbino, Università degli Studi di Urbino.

Said, E. (1991). Orientalismo. Torino, Bollati Boringhieri.

Said, E. (1998). Cultura e imperialismo. Letteratura e consenso nel progetto coloniale dell'Occidente. Roma, Gamberetti.

Sebag, P. (1998). Tunis: Histoire d'une ville. Paris, L'Harmattan.

Tumino, R. (2013). Guglielmo Collotti. In Dizionario Biografico dell'Educazione 1800-2000, (vol. I, A-K, 372-373). Milano, Editrice Bibliografica.

Ximenes E. (1885). Epistolario di Giuseppe Garibaldi con documenti e lettere inedite (1836-1882). Vol. II. Milano, Alfredo Brigola.

\section{Fonti archivistiche}

Archives Nationales de Tunisie, Correspondance $d u$ Royaume de Tunis et du Royaume de Sardaigne, Correspondance de 1798 à 1828, Dos. 612, Doc. 10. 